\title{
Usability Study on Healthy Food Restaurant Finder
}

\section{Lau Pei Shin ${ }^{1}$, Nooraini Yusoff ${ }^{2 *}$, Shahzad Qaiser ${ }^{3}$, Mohd Saberi Mohamad ${ }^{4}$, Khairul Nizar Syazwan WSW}

${ }^{1}$ School of Computing, UUM College of Arts and Sciences, Universiti Utara Malaysia, 06010 UUM Sintok, Kedah, Malaysia

${ }^{2,4,5}$ Institute for Artificial Intelligence and Big Data (AIBIG), Universiti Malaysia Kelantan, City Campus, 16100 Kota Bharu, Kelantan, Malaysia

${ }^{3}$ Department of Computer Science, Capital University of Science and Technology (CUST) Islamabad Expressway, Kahuta Road Zone-V, Islamabad, Pakistan

Corresponding author: ${ }^{2 *}$ nooraini.y@umk.edu.my

Article History: Received: 10 November 2020; Revised: 12 January 2021; Accepted: 27 January 2021;

Published online: 05 April 2021

Abstract: Good nutrition is an important part of leading a healthy lifestyle. However, it is not easy to maintain a wellbalanced diet every day especially when we are eating in a restaurant. Besides, people nowadays have low awareness about the food ingredients and it is not easy to measure the nutrition when people are preparing their own food or having meal outside. In addition, application such as Food panda, Grab Food and Facebook only recommend users about the best restaurant but do not include information about healthy eating. Therefore, the purpose of this project namely Healthy Eat Out application, is to help people to find the nearby healthy food. The app is developed using Android Studio via prototyping methodology to achieve user's requirements. This application is able to give information about food ingredients to users including how much calories the food contain. This can help to extend the knowledge of user about healthy food and create awareness of people when choosing eating outside.

Keywords: healthy, healthy food, healthy food restaurant, healthy eat out

\section{Introduction}

Food is essential for every human being. A study revealed that more than 80,000 new cancer cases are estimated to be associated with suboptimal diet among US adults in 2015 [1]. Such intake can also cause chronic illness therefore; people need to take healthy food because good nutrition is an important part of leading a healthy lifestyle [2]. There are many restaurants that serve healthy options such as vegetable sides or baked potatoes instead of French fries or water instead of soda [3,7]. However, the people always have limited time to explore the new restaurants. Therefore, Healthy Eat Out is an application to help people to find healthy food restaurant in Kedah state, Malaysia. Healthy Eat Out application has its function including search restaurant, view restaurant, view food and ingredients for the user to find the healthy food restaurant easily. Users can know directly about the healthy food of each restaurant. This will solve the problem of existing application and increase the awareness of people about the important of taking healthy food.

\section{Background and Related Studies}

Based on a previous national nutrition survey conducted in Malaysia, Malaysian adults had a daily median energy intake of $1466 \mathrm{kcal} /$ day or $64 \%$ of the Malaysian Recommended Nutrient Intakes (RNI), with men (1489 $\mathrm{kcal} / \mathrm{day})$ reporting a higher median energy intake than women (1445 kcal/day) [3]. The study observed that energy intake among the Malaysian population falls below the recommended intake level. Even for people at a healthy weight, a poor diet is associated with major health risks that can cause illness such as heart disease and cancer. In addition, poor diet quality is the primary risk factor responsible for the increased prevalence of obesity [4].

Therefore, people need to concern about the healthy level of food they take every single day such as how many calories the food contain or what is the sugar level they should observe for each day. Mostly, people are unclear about to whom the calorie and nutrient values applied and how this information was relevant to their own dietary requirements [5-6]. It is hard to measure the nutrition when people are preparing their own food or having meal outside.

Based on Google Play Store, mobile application such as Healthy Out and food Tripping can be described as application that help users to find the nearby healthy food restaurant. This application can also give information about the nutrition for dishes including calories and sugar. However, the application is not available in Malaysia. Besides, the application such as Facebook which can be accessed by Malaysian only suggests the best restaurant 
but does not consider on the healthy level of food provided [8]. Table 1 below shows the features of some existing applications.

Table 1. Example of Existing Application to Search For Healthy Food Restaurants

\begin{tabular}{|l|l|}
\hline Applications & Features \\
\hline Burpple & Guides users to good food and best restaurant. \\
\hline Food Tripping & $\begin{array}{l}\text { Discovers healthy, sustainable food options near you or in the place you're traveling } \\
\text { to. }\end{array}$ \\
\hline Healthy Out & Finds healthy restaurant nearby. \\
\hline Zomato & $\begin{array}{l}\text { It is a restaurant finder. Users can view menus, pictures, phone numbers, directions, } \\
\text { user reviews, and all the other information. }\end{array}$ \\
\hline Open Rice & $\begin{array}{l}\text { Recommends nearby restaurant for the user. Users can also get the information such } \\
\text { as location, price of food and operation hour from the application. }\end{array}$ \\
\hline
\end{tabular}

Therefore, for this project the Healthy Eat Out application is developed for the users in Kedah, Malaysia as a case study. This application is to help users to search nearby healthy food restaurant. It also provides the information on healthy food such as price and ingredients to users. Users can choose the suitable restaurant for them to eat out. It is easier and helpful because they do not need to explore all the restaurant to find the healthy food in Kedah.

\section{Methodology of The Study}

The methodology for this project follows the prototyping approach. According to International Software Testing Qualifications Board (ISTQB), the basic idea in Prototype model is that: "Instead of freezing the requirements before a design or coding can proceed, a throwaway prototype is built to understand the requirements. Prototyping is an attractive idea for complicated and large systems for which there is no manual process or existing system to help determine the requirements." A prototype model can be implemented as in Figure 1 shown below:

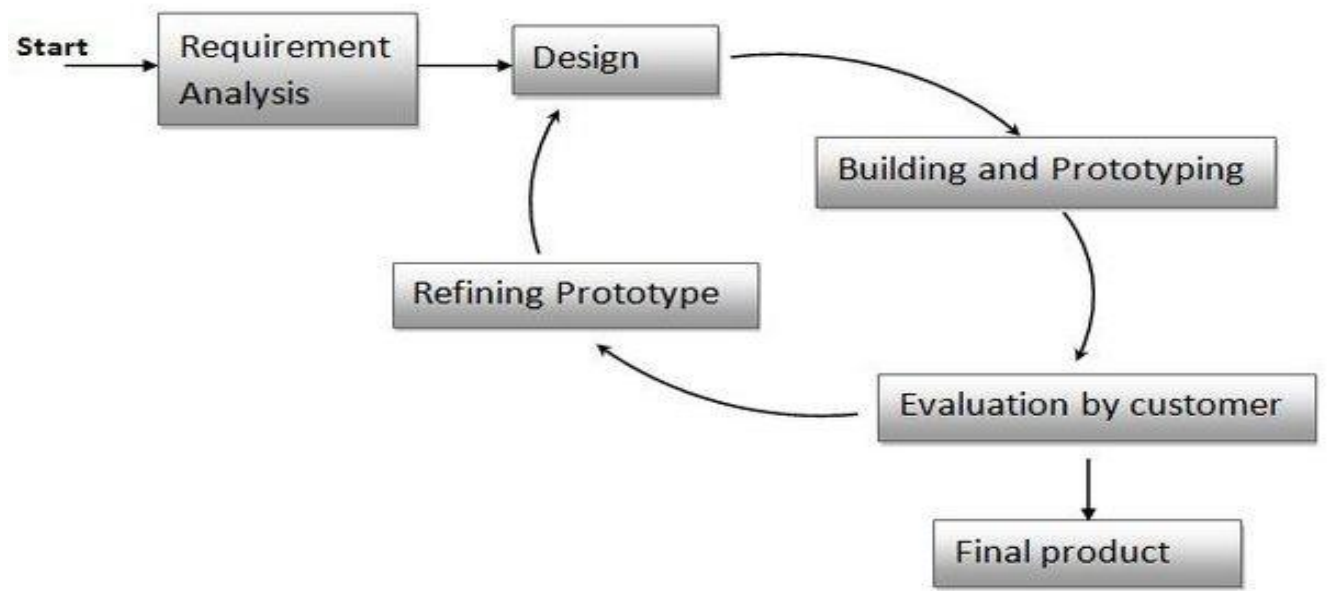

Figure 1. The phases of prototyping

The requirements of Healthy Eat Out application are gathered through a series of interviews with a number of potential users. Next, based on the gathered and analysed requirements, a preliminary design for the app is produced. This followed by the building and prototyping phase, in which the first prototype of Healthy Eat Out is developed based on the preliminary design. Initial version of the app represents an approximation of the characteristics of the final project.

In the evaluation phase, the users thoroughly evaluate the first prototype, noting about the strengths and weaknesses such as what need to be added or removed to improve the performance of application. The evaluation results and remarks from the users are collected and analysed. The findings provide input to the refinement of initial prototype. The steps are repeated from prototype design to refinement until the users' satisfaction has been achieved, hence that the prototype represents the desired final product. Lastly, in the final product phase, the final version of the Healthy Eat Out application is constructed. The final system is also 
thoroughly evaluated and tested. Furthermore, routine maintenance is to be carried out on a continuing basis to prevent large-scale failures and to minimize downtime.

\section{Design and Development to Healthy Eat Out}

This section is discussed in twofold. Firstly, the requirements of Healthy Eat Out application are discussed, followed by the description of application prototype development.

Table 2. List of Requirements For Healthy Eat Out Application

\begin{tabular}{|c|c|c|}
\hline ID & Description & Priority \\
\hline 1 & Login & \\
\hline 1.1 & Restaurant can login as restaurant & High \\
\hline 1.2 & User can login as customer & Medium \\
\hline 1.3 & Restaurant or customer can enter Email and Password to login. & High \\
\hline 2 & Sign Up & \\
\hline 2.1 & $\begin{array}{l}\text { For Restaurant and customer who does not have the account of HEO, } \\
\text { they can fill their information and submit it to register a new account of } \\
\text { HEO }\end{array}$ & High \\
\hline 3 & View Food & \\
\hline 3.1 & Customer can view list of healthy food of restaurant & High \\
\hline 3.2 & Customer can view the photo of the food & High \\
\hline 3.3 & Customer can view the price of food & Medium \\
\hline 3.4 & Customer can view ingredients of food. & High \\
\hline 4 & Add Food & \\
\hline 4.1 & Restaurant can add restaurant's name & Medium \\
\hline 4.2 & Restaurant can add restaurant's location & Medium \\
\hline 4.3 & Restaurant can add healthy food of restaurant & High \\
\hline 4.4 & Restaurant can add the photo of healthy food & High \\
\hline 4.5 & Restaurant can add ingredients of healthy food & High \\
\hline 4.6 & Restaurant can add other description of healthy food & Medium \\
\hline 5 & Manage Food & \\
\hline 5.1 & Restaurant can delete the healthy food & High \\
\hline
\end{tabular}

Figure 2 illustrates the use case diagram and the communications between the use cases and the actor for the Healthy Eat Out that can be used to allow users to search for healthy food restaurants. Five major use cases are login, sign up, view food, add food and manage food. 


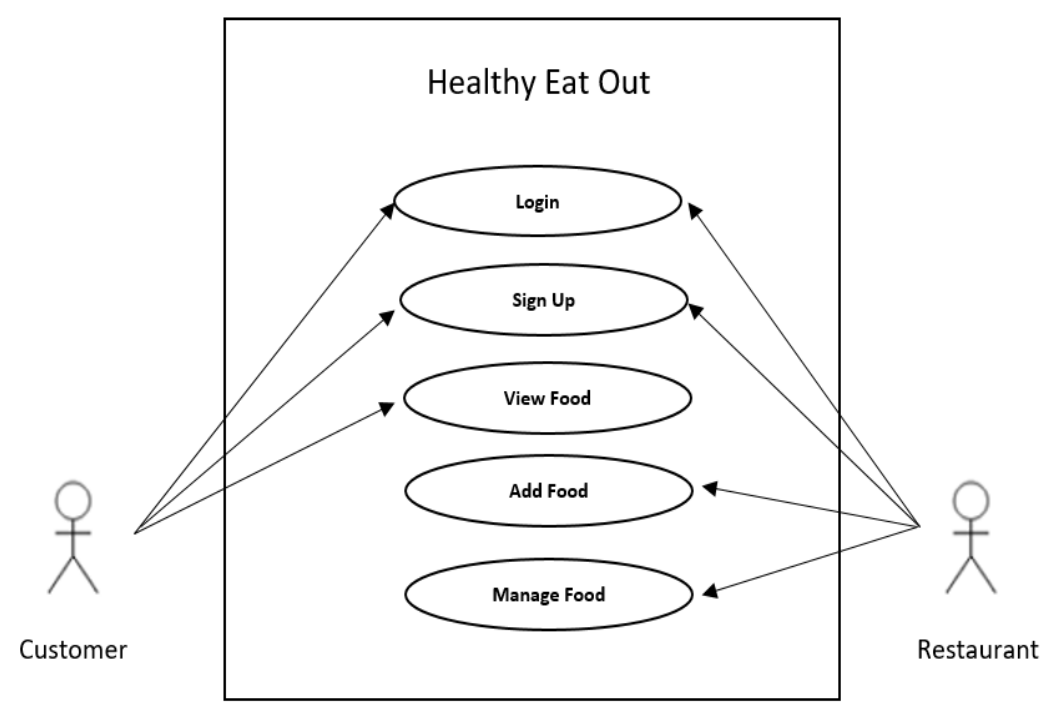

Figure 2. The use case diagram of Healthy Eat Out

Dynamic behaviour of the app is shown using a case diagram. Example of the operations involved in using the Healthy Eat Out application are illustrated in the activity diagram in Figure 3 and 4.

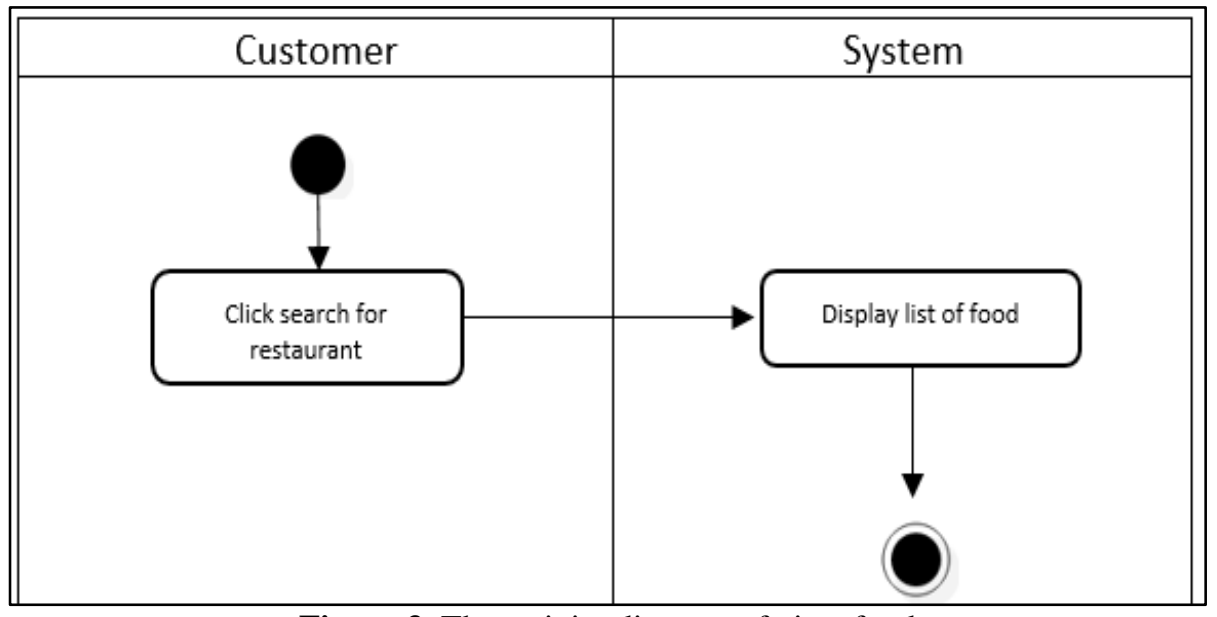

Figure 3. The activity diagram of view food

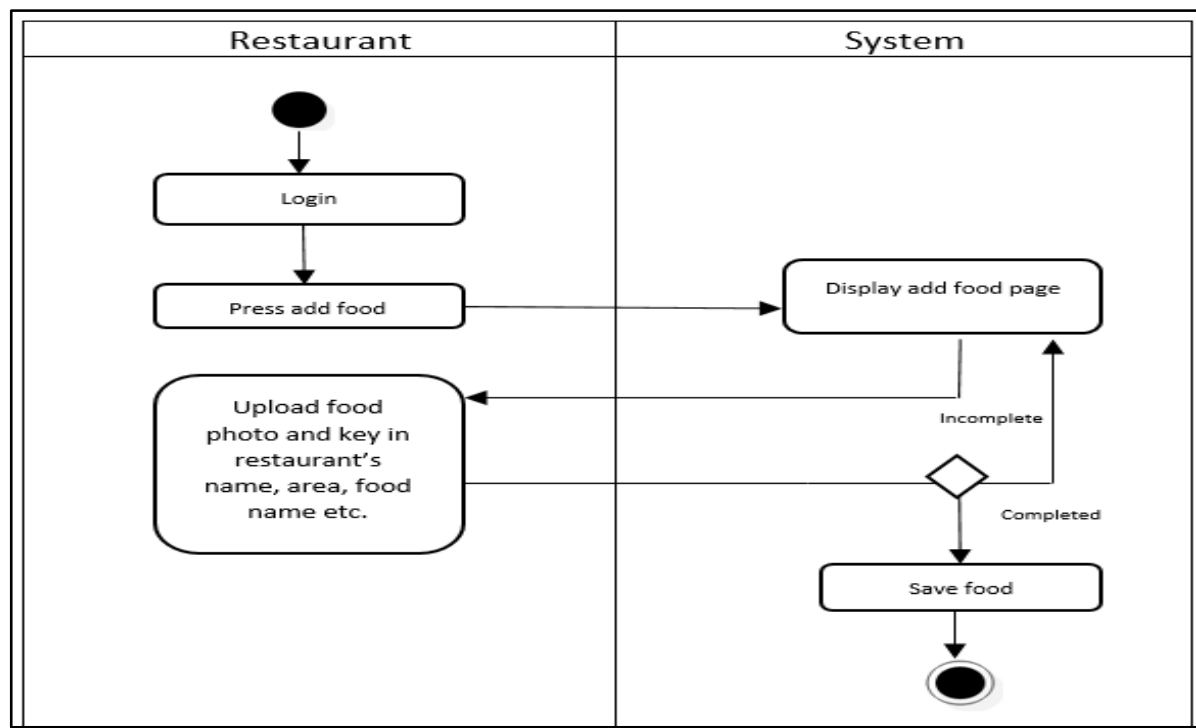

Figure 4. The activity diagram of add food 
The structural components of Healthy Eat Out application are represented in a class diagram as depicted in Figure 5 .

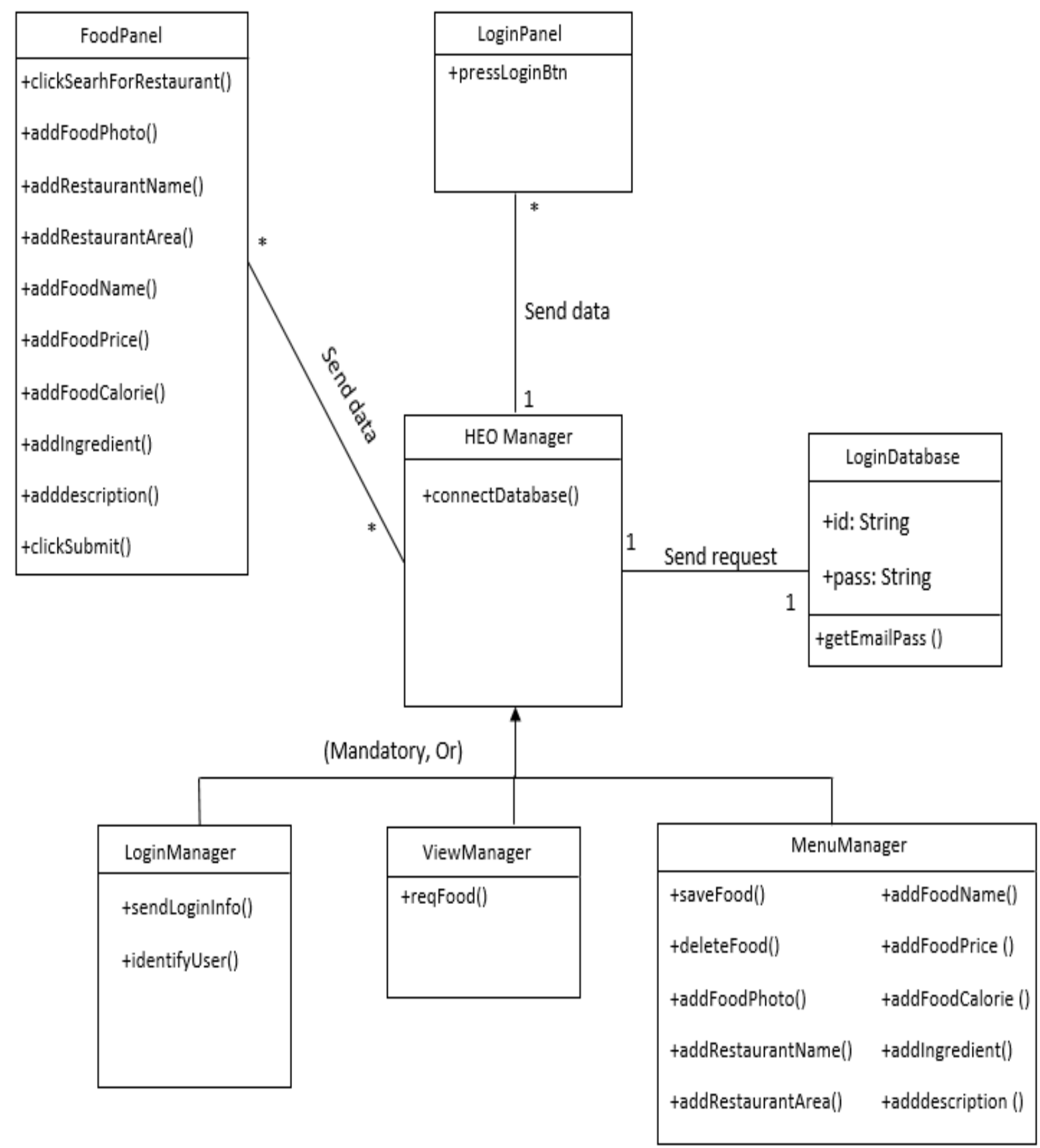

Figure 5. The class diagram of Healthy Eat Out

\section{The Healthy Eat Out Prototype Development}

In this phase, the prototype of Healthy Eat Out was developed. It complies all the requirements described in the previous subsection. The prototype was developed in the Android Studio integrated development environment (IDE). Meanwhile, the crucial functions including user authentication, and database for data storage, were created using Firebase development platform. Screenshots in Figures 6 and 7 show the selected interfaces of Healthy Eat Out. 


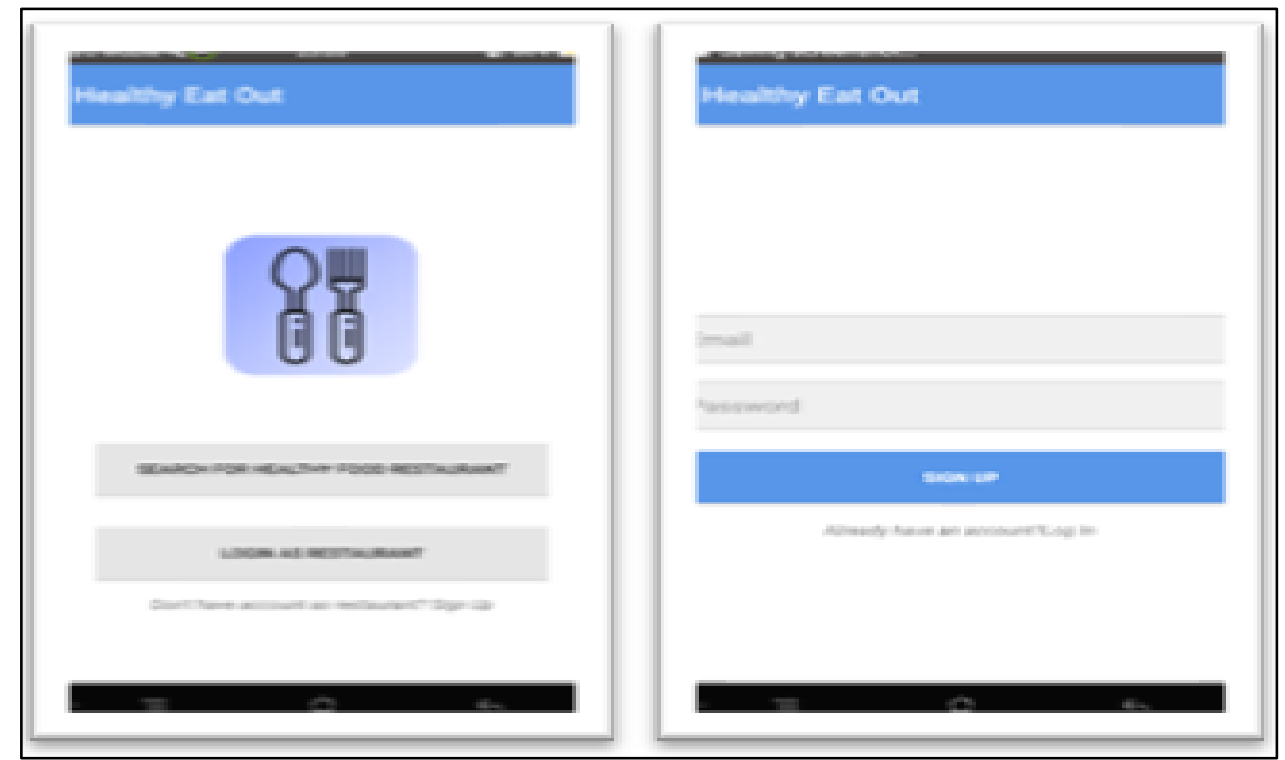

Figure 6. The interface of main page (left) and login (right)

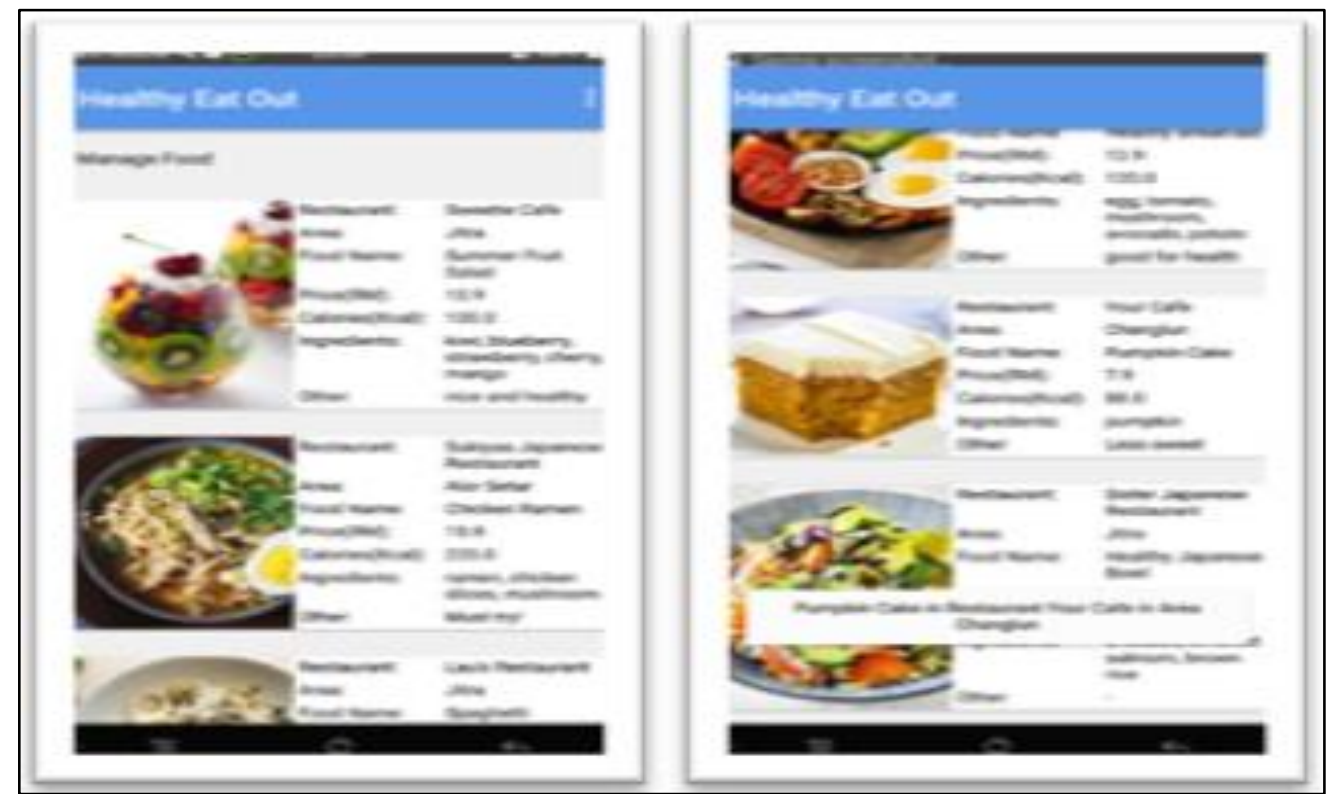

Figure 7. The interface of view food

\section{Evaluation of healthy eat out}

\section{A. The Evaluation Setting}

For evaluation of usability acceptance, user feedbacks were collected from 30 respondents consisting of Universiti Utara Malaysia (UUM) students. The respondents were approached randomly at UUM and participated in the study on a voluntary basis. The instruments used for the evaluation were the Healthy Eat Out application and post-task questionnaire. The questionnaire was filled up by the respondents. The questionnaire consisted of respondent demography and background information, and application usability and the feedback or suggestion for improving the application.

\section{B. The Respondents' Demographic Information}

Analysis of the respondents' demographic information revealed that $67 \%$ of them is female and $33 \%$ of them is male. 


\section{Gender}

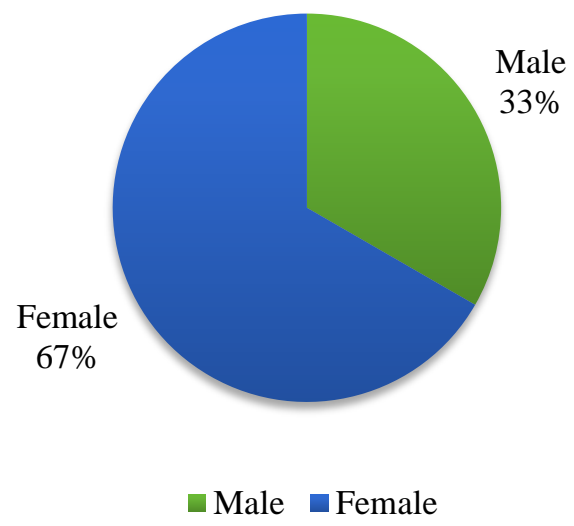

Figure 8. The analysis of respondents' demographic information

\section{The Usability of Healthy Eat Out}

The sections measure the respondents' perception towards Healthy Eat Out functionality, user interface and user acceptance. Figures 9 - 11 summarise the frequency and average of the responses. The respondents rated four or five of the post-task scales for three aspects of the usability (i.e. functionality, user interface and user acceptance. None of the respondents rated one or two. Only a few rated three.

\section{Functionality}

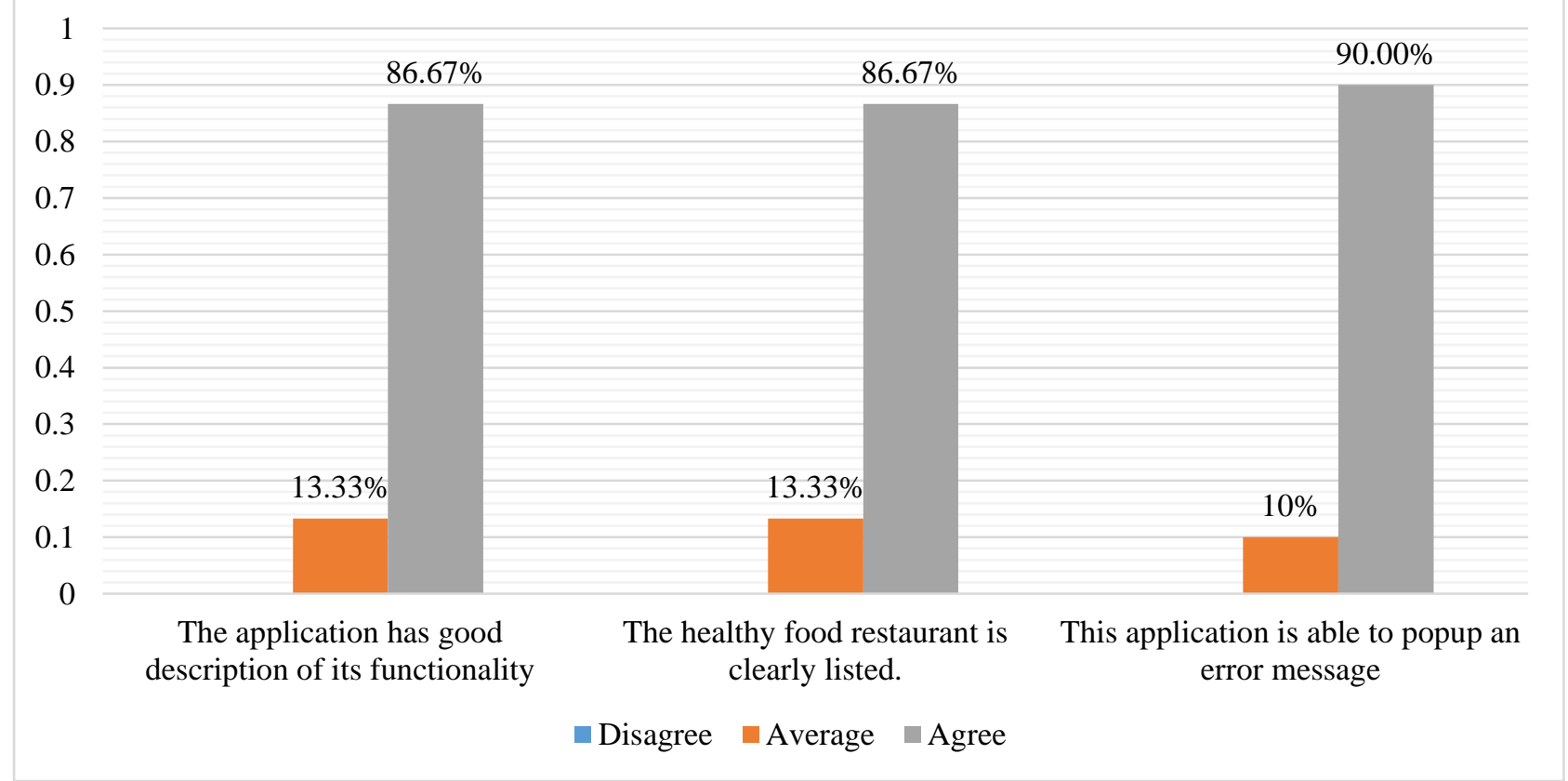

Figure 9. The respondents' responses on the functionality of Healthy Eat Out 


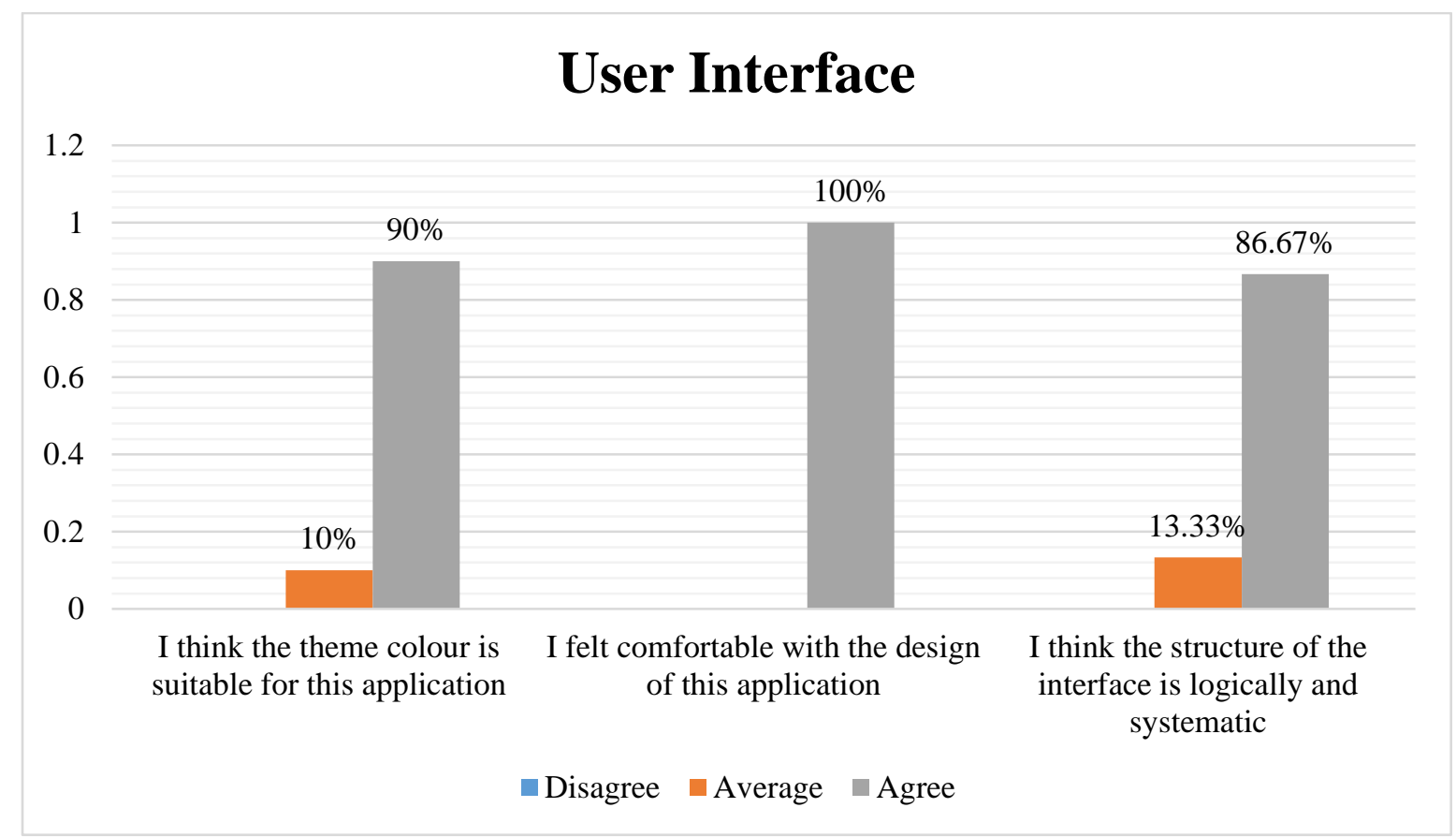

Figure 10. The respondents' responses on the user interface of Healthy Eat Out

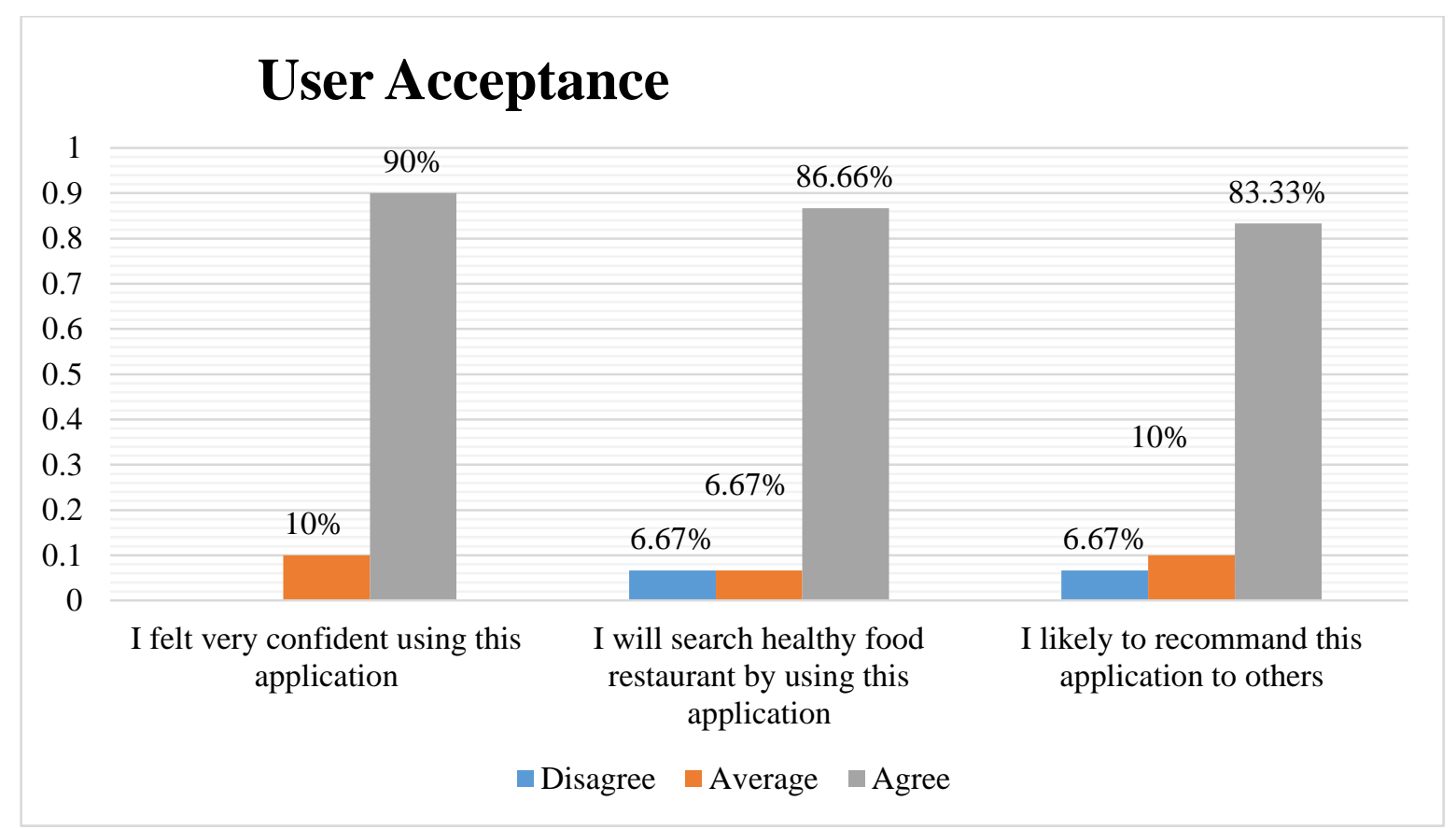

Figure 11. The respondents' responses on the user acceptance of Healthy Eat Out

The majority of respondents agree on the good functionality of Healthy Eat Out application. There were 26 of respondents agreed that the application has good description of its functionality. Besides, there were also 26 of respondents agreed that the healthy food restaurant is clearly listed in the application. In addition, there were 27 of respondents agreed that the application is able to pop up an error message.

Furthermore, there were majority of respondents agreed on the good user interface of the Healthy Eat Out application. There were 27 of respondents agreed that the theme colour of the application is suitable. Besides, all of the respondents felt comfortable with the design of the application. Furthermore, there were 26 of respondents thought that the structure of the interface is logically and systematic.

In addition, there were majority of respondents agreed on the Healthy Eat Out user acceptance features. There were 27 of respondents felt very confident using the application. Besides, there were 26 of respondents agreed 
that they would search healthy food restaurant by using Healthy Eat Out application. Furthermore, there were 25 of respondents were likely to recommend this application to others.

\section{Conclusion and future works}

According to the feedback collected, most of the respondents think that the application is simple and easy to use. Some of the respondents think that this application should have google maps so that the user can search for the location of healthy food restaurant directly. Besides, some of the respondents suggested that it is better if the application could show the picture of the restaurant and more pictures about the healthy food.

Besides, some of them also gave feedback about the design or interface of the application. Some of the respondents think that the application can be more colourful to attract the users. Generally, majority of the respondents were satisfied and comfortable with the developed Healthy Eat Out application.

More research can be carried out in future to integrate a recommender system in this application so that it can offer personalized suggestions to the users for healthy eating [9]. Secondly, the usability study can be improved by using various methods such as New Fuzzy Kano Method [10].

\section{Acknowledgment}

The authors wish to thank all the respondents of the usability study conducted.

\section{References}

1. Fang Fang Zhang, MD, PhD Frederick Cudhea, PhDZhilei Shan, MD, PhD Dominique S Michaud, ScDFumiaki Imamura, PhD Heesun Eom Mengyuan Ruan, MSColin D Rehm, PhD Junxiu Liu, PhD Mengxi Du, MS David Kim, PhDLauren Lizewski, MPH Parke Wilde, PhD Dariush Mozaffarian, MD, DrPH (2019). Preventable Cancer Burden Associated with Poor Diet in the United States. Retrieved from https://academic.oup.com/jncics/advancearticle/doi/10.1093/jncics/pkz034/5492023?searchresult=1

2. Denise de Ridder, Floor Kroese, Catharine Evers, Marieke Adriaanse \& Marleen Gillebaart (2017) Healthy diet: Health impact, prevalence, correlates, and interventions, Psychology \& Health, 32:8, 907-941, DOI: 10.1080/08870446.2017.1316849

3. Megan A. Moreno, MD, MSEd, MPH; Fred Furtner; Frederick P. Rivara, MD, MPH(2013).Eating out at restaurants with children and teens. Retrieved from https://jamanetwork.com/journals/jamapediatrics/fullarticle/1555830

4. Institute for Public Health (IPH). National Health and Morbidity Survey 2014: Malaysian Adults Nutrition Survey (MANS). Vol. II: Survey Findings. Kuala Lumpur: Ministry of Health Malaysia; 2014. ISBN 978-983-2387-16-9

5. Swinburn BA, Caterson I, Seidell J, James W. Diet, nutrition and the prevention of excess weight gain and obesity. Public Health Nutr. 2007; 7: 123-146.

6. Taher, A. K., Evans, N. \& Evans, C. E. (2018). The cross-sectional relationships between consumption of takeaway food, eating meals outside the home and diet quality in British adolescents. Public Health Nutrition, 22(1), 63-73.

7. Fagerstrøm, A., Richartz, P., Pawar, S., Larsen, N. M., Sigurdsson, V., \& Eriksson, N. (2019). The relative importance of healthy food labels when shopping for groceries online. Procedia Computer Science, 164, 538-545.

8. Samoggia, A., \& Riedel, B. (2020). Assessment of nutrition-focused mobile apps' influence on consumers' healthy food behaviour and nutrition knowledge. Food Research International, 128, 108766.

9. Trang Tran, T.N., Atas, M., Felfernig, A. et al. An overview of recommender systems in the healthy food domain. J Intell Inf Syst 50, 501-526 (2018). https://doi.org/10.1007/s10844-0170469-0

10. Taimouri, A., Emamisaleh, K., \& Mohammadi, D. (2019). Assessing the Usability of Online Food Ordering Websites Using a New Fuzzy Kano Method: Implications for Improvement. International Journal of Business and Management, 14(10). 